Pacific Journal of Mathematic 


\title{
ON A BOUNDARY PROPERTY OF PRINCIPAL FUNCTIONS
}

\author{
MineKo Watanabe
}

\begin{abstract}
A behavior of $(P) L_{1}$-principal functions on some compactifications of a Riemann surface is studied. The main result in this paper is that a $(P) L_{1}$-principal function is extended almost everywhere continuously to some compactifications and the extention is almost everywhere constant on each part of $P$. If the genus of the surface is finite and $P$ is the canonical $\widetilde{P},(\widetilde{P}) L_{1}$-principal function can be extended continuously to the Kerékjártó-Stoilow compactification.
\end{abstract}

The method of normal operators on open Riemann surfaces was developed by Sario [10] and others, and they established the existence theorems of harmonic functions with given singularities and prescribed modes of behavior near the ideal boundary. Especially, principal functions constructed by principal operators have been taken up by several authors, and many interesting results have been proved. Kusunoki [4] introduced the notion of canonical potentials and canonical differentials, and developed the theory of Abelian integrals on open Riemann surfaces. This canonical potential is readily shown to be $(\widetilde{P}) L_{1}$-principal function corresponding to the canonical partition of the boundary, if it is single-valued (paragraph 3). On a compact bordered surface, a $(P) L_{1}$-principal function is defined by the property that it is constant on each part of partition $P$ of the boundary and has zero flux over each $P$-dividing cycle. The main purpose of the present paper is to study such property of $(P) L_{1}$-principal functions on the boundary of arbitrary open Riemann surfaces.

Nakai and Sario [8] showed that, in the case of identity partition, corresponding $L_{1}$-principal function can be extended finitely continuously and it is almost everywhere constant on the Royden boundary, and this property and vanishing of flux over the boundary characterize $L_{1}$-principal functions corresponding to the identity partition. Kusunoki [5] proved that a canonical potential on a Riemann surface has a constant value quasi-everywhere on each connected component of the Kuramochi boundary. We shall show in the present paper that a $(P) L_{1}$-principal function can be extended almost everywhere continuously so that the extention is almost everywhere constant on each part of a given regular partition $P$ of the boundary, where compactification of the Riemann surface may be of Martin, Kuramochi, Royden, Wiener, or a $Q$-compactification, denoting by $Q$ any sublattice of $H P$ which contains constant (Theorem 1). In the case of Martin compactification, each polar set on the boundary is 
also of harmonic measure zero. In the case of Kuramochi compactification, the condition 'almost everywhere' can be strengthened to 'quasi-everywhere'. Further, if the set of constant values of the extended function on the parts of $P$ satisfies a certain condition, this property, vanihing of the flux over any $P$-dividing cycle and Dirichlet integrability on a boundary neighborhood characterize the $(P) L_{1}$ principal functions (Theorem 2).

In the case of Riemann surfaces of finite genus, a $(\widetilde{P}) L_{1}$-principal function corresponding to the canonical partition $\widetilde{P}$ is everywhere constant on each part of $\widetilde{P}$ (Theorem 3). However, we can show by an example that this condition is not sufficient to characterize the $(\widetilde{P}) L_{1}$-principal functions on Riemann surfaces of finite genus.

1. Let $R$ be an open Riemann surface and let us denote by $P$ a regular partition of the ideal boundary of $R$ (Ahlfors-Sario [1]) and by $I$ the identity partition. Let $U$ be a boundary neighborhood such that $R-\bar{U}$ is a regular region, and $\Omega$ a canonical region containing $\overline{R-U}$. Let us also denote by the same $P$ the partition of the boundary $\partial \Omega$ of $\Omega$ which is induced by the original $P$. For given singularities $s$ in $R-\bar{U}$ with vanishing flux on $R$, there corresponds a $(P) L_{1}$-principal function $f_{P \Omega}$ on $\Omega$. This $f_{P \Omega}$ is defined by the following conditions:

(i) $f_{P \Omega}$ has the singularities $s$.

(ii) $f_{P Q}$ is constant on each part of $P$ on $\partial \Omega$.

(iii) the flux of $f_{P \Omega}$ vanishes over any part of $P . \quad B y$ (ii) and (iii), we have

$$
\left\|d f_{P \Omega}\right\|_{\Omega \cap \bar{U}}=-\int_{\partial U} f_{P \Omega} d f_{P \Omega}^{*}<\infty .
$$

According to Rodin-Sario [9], the suitably normalized family $\left\{f_{P \Omega}\right\}_{\Omega}$ converges uniformly on a compact set to a $(P) L_{1}$-principal function $f_{P}$ on $R$ with the singularities $s$.

Let $G$ be a regular region containing all singular points of $f_{P}$, then

$$
\left\|d f_{P}\right\|_{R-\bar{G}}=-\int_{\partial G} f_{P} d f_{P}^{*}<\infty
$$

(Ahlfors-Sario [1]). Therefore, for an arbitrary given positive $\varepsilon$, we can choose a sufficiently large compact set $K$ with a smooth boundary so that

$$
\left\|d f_{P}\right\|_{R-K}<\varepsilon
$$

and we can find $\Omega$ so large that 


$$
\left\|d f_{P}-d f_{P \Omega}\right\|_{K}<\varepsilon \text { and }\left|\int_{\partial K} f_{P} d f_{P}^{*}-\int_{\partial K} f_{P \Omega} d f_{P \Omega}^{*}\right|<\varepsilon .
$$

Then we have

$$
\begin{aligned}
\left\|d\left(f_{P}-f_{P \Omega}\right)\right\|_{\Omega} & =\left\|d\left(f_{P}-f_{P \Omega}\right)\right\|_{K}+\left\|d\left(f_{P}-f_{P \Omega}\right)\right\|_{\Omega-K} \\
& \leqq\left\|d\left(f_{P}-f_{P \Omega}\right)\right\|_{K}+\left\|d f_{P}\right\|_{R-K}+\left\|d f_{P \Omega}\right\|_{\Omega-K} \\
& <4 \varepsilon
\end{aligned}
$$

because

$$
\left\|d f_{P \Omega}\right\|_{\Omega-K}=\left|\int_{\partial K} f_{P \Omega} d f_{P \Omega}^{*}\right|<\left|\int_{\partial K} f_{P} d f_{P}^{*}\right|+\varepsilon=\left\|d f_{P}\right\|_{P-K}+\varepsilon .
$$

Thus we get

LeMma 1. $A(P) L_{1}$-principal function $f_{P}$ can be approximated in norm by principal functions $\left\{f_{P \Omega}\right\}_{\varrho}$ on canonical regions $\Omega$.

The same assertion can be proved quite analogously for an $L_{0}$-principal function too.

2. By Lemma 1 we readily see

Lemma 2. For any regular partition $P$ of the ideal boundary, we have

$$
f_{P}=f_{I}+h_{P}
$$

on $R$, where $d h_{P} \in(P) \Gamma_{h m}$.

Here $(P) \Gamma_{h m}$ is the Hilbert space spanned by the differentials of harmonic measures associated with $P$-dividing cycles. If $\gamma_{P}$ is a $P$-dividing cycle, $\gamma_{P}$ divides a sufficiently large $\Omega$ into two regions $\Omega_{1}$ and $\Omega_{2}$. Let $w_{\Omega}$ be a harmonic measure which is 1 on $\partial \Omega_{1}-\gamma_{P}$ and 0 on $\partial \Omega_{2}-\gamma_{P}$. Then $w_{\gamma}=\lim _{\Omega \rightarrow R} w_{\Omega}$ is a harmonic measure associated with $\gamma_{P}$.

On a compact bordered surface $\bar{\Omega}$, it is obvious that

$$
d\left(f_{P \Omega}-f_{I \Omega}\right)=d h_{P \Omega} \in(P) \Gamma_{h m}(\Omega) .
$$

We have

$$
\left\|d\left(h_{P}-h_{P \Omega}\right)\right\|_{\Omega} \leqq\left\|d\left(f_{P}-f_{P \Omega}\right)\right\|_{\Omega}+\left\|d\left(f_{I}-f_{I \Omega}\right)\right\|_{\Omega} \rightarrow 0 \text { as } \Omega \rightarrow R .
$$

Therefore $d h_{P} \in(P) \Gamma_{h m}$.

Let us remark that if $R \in 0_{H D},(P) \Gamma_{h m} \subset \Gamma_{h e}=\{0\}$, and therefore $(P) L_{1}$-principal functions coincide each other for all $P$. 
Lemma 3. An (I) $L_{1}$-principal function $f_{I}$ has the following representation on a boundary neighborhood $U$.

$$
f_{I}=r_{0}+c u
$$

where $c$ is a constant, $u$ is a harmonic measure of the ideal boundary with respect to $U$, and $r_{0}$ is a harmonic function on $U$ satisfying

$$
c_{1}(1-u) \leqq r_{0} \leqq c_{2}(1-u)
$$

with suitable constants $c_{1}$ and $c_{2}$.

Proof. By the definition of $(I) L_{1}$ on $R, f_{I}$ is a limit function of $g_{\rho}$ as $\Omega$ tends to $R$, where $g_{\Omega}$ satisfies

(i) $f_{I}=g_{\Omega}$ on $\partial U$.

(ii) $g_{\Omega}$ is constant on $\partial \Omega$.

(iii) $\int_{\partial \Omega} d g_{\Omega}^{*}=0$.

Let us denote by $r_{0 \Omega}$ the harmonic function on $\Omega \cap U$ which is equal to $f_{1}$ on $\partial U$ and 0 on $\partial \Omega$. Then $\lim _{\Omega \rightarrow R} r_{0 \Omega}=r_{0}$ exists and it is harmonic on $U$. The function $g_{\Omega}-r_{0 \Omega}$ is 0 on $\partial U$ and constant, say $c_{\Omega}$, on $\partial \Omega$. Then it is equal to $c_{\Omega} u_{\Omega}$, where $u_{\Omega}$ is a harmonic measure of $\partial \Omega$ with respect to $\Omega \cap U$. We have

$$
\lim _{\Omega \rightarrow R}\left(g_{\Omega}-r_{0 \Omega}\right)=f_{I}-r_{0}=\lim _{\Omega \rightarrow R} c_{\Omega} u_{\Omega}
$$

and

$$
\lim _{\Omega \rightarrow R} u_{\Omega}=u \text {. }
$$

Hence $c=\lim _{\Omega \rightarrow R} c_{\Omega}$ exists and is equal to $\left(\lim c_{\Omega} u_{\Omega}\right) / \lim u_{\Omega}$. Further we have

$$
\left(\min _{\partial U} f_{I}\right)(1-u) \leqq r_{0} \leqq\left(\max _{\partial U} f_{I}\right)(1-u) \text { on } U \text {. }
$$

3. Lemmas 2 and 3 show that in order to know the behavior of $(P) L_{1}$-principal functions on the boundary, it is enough to study that of harmonic measures.

Let us remind that a $Q$-compactification of $R$, where $Q$ is a class of continuous functions, is a compactification $R_{Q}^{*}$ of $R$, on which all functions of $Q$ can be extended continuously and the extended functions separate the points on $\Delta_{Q}=R_{Q}-R$. For each potential $q$, let $\delta_{p}$ be the set of points $b \in \Delta_{Q}$ such that

$$
\lim _{a \rightarrow b} q(a)=0 \text {, }
$$


and let $\delta_{Q}=\cap \delta_{q}$ where $q$ runs through the class of all potentials. We call $\delta_{Q}$ a harmonic boundary of $R_{Q}^{*}$. If $R_{Q}^{*}$ is a resolutive compactification, $\delta_{Q}$ is a carrier of harmonic measure $\omega$ on $\Delta_{Q}$, and therefore $\omega\left(\Delta_{Q}-\delta_{Q}\right)=0$ (p. 92, Constantinescu-Cornea [2]).

Let us denote by $D$ and $W$ the class of Dirichlet functions and Wiener functions respectively, then by definition $R_{D}^{*}$ is a Royden compactification and $R_{W}^{*}$ is a Wiener compactification. Of course, we have $D \subset W$. Let $Y$ be a sublattice of $H P$ which contains constant, then $Y \subset W$. If $Y \subset W, R_{Y}^{*}$ is resolutive (p. 99, ConstantinescuCornea [2]). Further all points of $\delta_{D}, \delta_{W}$ and $\delta_{Y}$ are regular (p. 101, [2]).

Let $R_{Q}^{*}$ be a resolutive compactification. A $P$-dividing cycle $\gamma_{P}$ divides $R$ into two parts $R_{1}$ and $R_{2}$. Let $\Delta_{1}=\Delta_{Q} \cap \bar{R}_{1}$ and $\Delta_{2}=\Delta_{Q} \cap \bar{R}_{2}$, then harmonic measure associated with $\gamma_{P}$ are given by

$$
w_{\gamma_{P}}=\int_{\Lambda_{1}} h d \omega, \quad \widetilde{w}_{\gamma_{P}}=1-w_{\gamma_{P}}
$$

where $h$ is a function on $\Delta_{Q}$ which is 1 on $\Delta_{1}$ and 0 on $\Delta_{2}$. Hence, if all points of $\delta_{Q}$ are regular, $w_{\gamma_{P}}=1$ on $\delta_{Q} \cap \Delta_{1}$ and 0 on $\delta_{Q} \cap \Delta_{2}$.

Lemma 4. An integral of any element of $(P) \Gamma_{h m}$ is constant almost everywhere on each part of partition $P$, if the compactification is resolutive and all points of harmonic boundary are regular.

For the harmonic measure $u$ of the ideal boundary with respect to $U$, it is easily seen that $u=1$ on $\delta_{Q}$ under the same conditions.

In the case of Martin compactification, the set of points $b$ on the boundary such that

$$
\varlimsup_{a \rightarrow b} g\left(a, a_{0}\right)>0
$$

is polar, where $g\left(a, a_{0}\right)$ is a Green function on the Riemann surface with a pole at $a_{0}$. Comparing with this Green function in a boundary neighborhood, we can show that $\lim _{a \rightarrow b} w_{\tau_{P}}(a)=0$ or 1 and $\lim _{a \rightarrow b} u(a)=$ 1 quasi-everywhere on the boundary.

If $R$ is hyperbolic, a function $f$ which is equal to $1-u$ on $U$ and $\equiv 1$ on $R-U$ is a Dirichlet potential on $R$, which is seen by the fact that the greatest harmonic minorant of $f$ vanishes identically. There is also a Dirichlet potential on $R$, which is equal to $w_{\gamma_{P}}$ on $R_{2}$ and $\equiv 0$ in a neighborhood of $\Delta_{1}$. On a Kuramochi compactification a Dirichlet function is a Dirichlet potential if and only if whose quasi-continuous extention vanishes quasi-everywhere on the boundary $\Delta$ (p. 193, Constantinescu-Cornea [2]). Hence we obtain the fact that 
an integral of any element of $(P) \Gamma_{h m}$ has a constant value quasieverywhere on each part of $P$ on $\Delta$, and $u=1$ quasi-everywhere on $\Delta$.

Thus by Lemmas 2 and 3 , we get

THEOREM 1. Suppose that a compactification $R^{*}$ of $R$ is anyone of Royden, Wiener or a Q-compactification where $Q$ is a sublattice of $H P$ which contains constant. Then a $(P) L_{1}$-pincipal function can be extended almost everywhere continuously on $R^{*}$ so that the extention is constant almost everywhere on each part of partition $P$. In the case of Martin or Kuramochi compactification, the condition, 'almost everywhere' can be replaced more restrictive 'quasi-everywhere'.

Let us notice that if the partition $P$ is canonical $\widetilde{P}$, a $(\widetilde{P}) L_{1}$ principal function is a single-valued canonical potential and vice versa. Indeed, if $f_{1}$ is a $(\widetilde{P}) L_{1}$-principal function with canonical $\widetilde{P}$, there is a single-valued canonical potential $g$ with the same singularities as $f_{1}$. On a sufficiently large $\Omega$ there are a $(\widetilde{P}) L_{1}$-principal function $f_{\Omega}$ and a single-valued potential $g_{\Omega}$ which satisfy

$$
\lim _{\Omega \rightarrow R}\left\|d f_{1}-d f_{\Omega}\right\|_{\Omega}=0, \quad \lim _{\Omega \rightarrow R}\left\|d g-d g_{\Omega}\right\|_{\Omega}=0 .
$$

On $\Omega$ we have

$$
d f_{\Omega}-d g_{\Omega} \in \Gamma_{h m}(\Omega) \cap \Gamma_{h s e}^{*}(\Omega)=\{0\},
$$

and therefore we get

$$
d f_{1}-d g \in \Gamma_{h m} \cap \Gamma_{h s e}^{*}=\{0\}
$$

on $R$.

4. Any principal function $f$, of $L_{1}$ or $L_{0}$, has a finite Dirichlet integral on a boundary neighborhood and satisfies

$$
\lim _{\Omega \rightarrow R} \int_{\partial \Omega} f d f^{*}=0 \text {. }
$$

Further, by Theorem 1 , we know that a $(P) L_{1}$-principal function $f_{P}$ has a following property $(\alpha)$.

$(\alpha) f_{P}$ can be extended on $R^{*}$ almost everywhere continuously so that it is constant almost everywhere on each part of $P$.

Now we consider under what restriction, the condition $(\alpha)$ and the following $(\beta)$ and $(\gamma)$ characterize $(P) L_{1}$-principal functions.

$(\beta)$ The Dirichlet integral taken over a boundary neighborhood is finite.

$(\gamma)$ The flux over each $P$-dividing cycle vanishes. 
If $R \in O_{K D}$, every real function $h$ which is harmonic except polar singularities and has the properties $(\beta)$ and $(\gamma)$ with canonical $\widetilde{P}$, is nothing but a $(\widetilde{P}) L_{1}$-principal function corresponding to the canonical $\widetilde{P}$. Indeed, if $f_{1}$ is a $(\widetilde{P}) L_{1}$-principal function with the same singularities as $h$, then

$$
d\left(f_{1}-h\right) \in \Gamma_{h e} \cap \Gamma_{h s e}^{*}=\{0\} .
$$

As a sufficient condition for that the three conditions $(\alpha),(\beta)$ and $(\gamma)$ characterize $(P) L_{1}$-principal functions, we can prove

THEOREM 2. Suppose that a compactification $R^{*}$ is one of Royden, Wiener, Martin, Kuramochi or a Q-compactification with a sublattice $Q$ of $H P$ containing constant, and a function $g$ which is harmonic except polar singularities, satisfies $(\alpha),(\beta)$ and $(\gamma)$. If the set of constant values which are taken by $g$ on parts of $P$ is discrete except the supremum and infimum, then $g$ is a $(P) L_{1}$ principal function.

Proof. Let $g$ be a function satisfying the assumption in the theorem, and $f_{I}$ a $(I) L_{1}$-principal function with the same singularities as $g$. Let $h=f_{I}-g$, then $d h \in \Gamma_{h e} \cap(P) \Gamma_{h s_{e}}^{*}$ and $h$ has the property $(\alpha)$. Let $c_{0}$ be one constant value of $h$ on a part of $P$ which is not the supremum nor infimum. We put the constant values of $h$ on the boundary in line as follows.

$$
\cdots<c^{(M)}<\cdots<c^{(2)}<c^{(1)}<c_{0}<c_{1}<c_{2}<\cdots<c_{N}<\cdots .
$$

Let $h \vee\left(c^{(M)}\right)=h_{M}$, then we have $h_{M I} \geqq h_{M+1}$ and

$$
\left\|d h_{M}\right\| \leqq\|d h\|_{h \geqq c(M)} \leqq\|d h\| \text {. }
$$

Let $h_{M} \wedge c_{v}=h_{M N}$, then $h_{M N} \leqq h_{M N+1}$, and

$$
\left\|d h_{M N_{N}}\right\| \leqq\left\|d h_{M}\right\|_{h_{M} \leqq c_{N}} \leqq\left\|d h_{M}\right\|,
$$

and it is readily seen that $d h_{M N} \in(P) \Gamma_{h m}$. By (2), a proper subsequence of $\left\{h_{M N}\right\}_{N}$ converges to $h_{M}$ in norm sense and therefore $d h_{M} \in(P) \Gamma_{h m}$. By (1), a proper subsequence of $\left\{h_{M}\right\}_{M}$ converges to $h$ in norm sense, and therefore $d h \in(P) \Gamma_{h m}$. Hence we get that $g\left(=f_{I}+h\right)$ is a $(P) L_{1}$ principal function.

5. Now we restrict $R$ to be of finite genus and the partition $\widetilde{P}$ to be canonical, and consider a compactification $R^{*}$ of type $S$. We say a compactification $R^{*}$ is of type $S$, if for any region $G^{*} \subset R^{*}$ whose boundary is in $R, G^{*}-\Delta$ is connected (Constantinescu-Cornea [2]). Let $f_{1}$ be a $(\widetilde{P}) L_{1}$-principal function. If a boundary component 
$\Delta_{e}$ of $R$ is weak, $f_{1}+i f_{1}^{*}$ and hence $f_{1}$ has a limit at $\Delta_{e}$, which is shown as Lemma 2 in Mori [7]. Though the $f_{1}$ is single-valued on $R$, the $f_{1}+i f_{1}^{*}$ may not be single-valued. But this function is semi-exact, and we can choose a boundary neighborhood on which a branch of $f_{1}+i f_{1}^{*}$ is single-valued. If $\Delta_{e}$ is not weak, we can show that a harmonic measure $u$ of the ideal boundary with respect to a boundary neighborhood can be extended continuously so that $u=1$ on $\Delta_{e}$. A harmonic measure $w_{\gamma}$ associated with a dividing $\gamma$ is also identically 1 or 0 on $\Delta_{e}$.

A dividing cycle $\gamma$ divides the boundary $\Delta$ into two parts $\Delta_{1}$ and $\Delta_{2}$, and a canonical region $\Omega$ into $\Omega_{1}$ and $\Omega_{2}$ if $\Omega$ is sufficiently large. Let $w_{\Omega}$ be a harmonic function on $\Omega$ which is 1 on $\partial \Omega_{1}-\gamma$ and 0 on $\partial \Omega_{2}-\gamma$, then $\lim _{\Omega \rightarrow R} w_{\Omega}=w_{\gamma}$. Since the genus of $R$ is finite, there is a planar neighborhood $U_{e}$ of $\Delta_{e}$, and a conformal mapping $\varphi$ of $U_{e}$ by which $\Delta_{e}$ corresponds to a continuum $\lambda^{*}$ on a plane. Then by a properly normalized slit mapping $\psi$ of the complement of $\lambda^{*}$, the image of $U_{e}$ can be mapped conformally on a region on a $z$-plane so that $\lambda^{*}$ corresponds to a segment $\lambda$ on the real axis which contains the origin as an interior point. Let $r$ be a positive number on $\lambda$, and denote by $K_{r}$ the disk $|z| \leqq r$. Take a parameter $t=\log z$ and consider a harmonic measure $\mu(t)$ of the image of the circle $|z|=r$, $0 \leqq \arg z<2 \pi$ with respect to the half plane $\operatorname{Re} t \leqq \log r$. The function $\mu(\log z)$ is harmonic in $0<|z| \leqq r$ and single-valued if $0 \leqq$ $\arg z<2 \pi$, and

$$
\mu(\log z) \leqq \frac{2}{\pi} \tan ^{-1}\left(\log \frac{r}{|z|}\right)^{-1}
$$

Further, for a sufficiently large $\Omega$, we have

$$
w_{\Omega}\left(\varphi^{-1}\left(\psi^{-1}(z)\right)\right)<\mu(\log z)
$$

which is seen by the maximum principle. Combining these two inequalities we get the above result.

Thus, by the use of Lemmas 2 and 3 we obtain that a $(\widetilde{P}) L_{1}$ principal function has a limit on each boundary component. Therefore we can extend the function to a Kerékjártó-Stoilow compactification. The fact that the extended function $f_{1}$ is continuous on $R^{*}$ is readily seen because each boundary component has a planar neighborhood and the $f_{1}+i f_{1}^{*}$ is conformal on the neighborhood.

THEOREM 3. If a Riemann surface is of finite genus, a $(\widetilde{P}) L_{1^{-}}$ principal function associated with the canonical partition $\widetilde{P}$ has a continuous extention on a Kerékjártó-Stoilow compactification. 
6. The converse of the theorem is not true, that is, there is a Riemann surface of finite genus with a function defined on it which can be extended continuously on a Kerékjártó-Stoilow compactification, but is not a $(\widetilde{P}) L_{1}$-principal function.

Let us consider a Riemann surface $R$ of finite genus which is not of class $O_{K D}$ but whose all boundary components are weak. The existence of such a Riemann surface was proved in Jurchescu [3]. An $L_{0}$-principal function $f_{0}$ is a limit of properly normalized family $\left\{f_{0 \Omega}\right\}_{\Omega}$, where each $f_{0 \Omega}$ is defined by the property that the normal derivative vanishes along $\partial \Omega$ (Rodin-Sario [9]). Obviously $f_{0}$ satisfies the conditions $(\beta)$ and $(\gamma)$. Moreover, $f_{0}$ has a limit at any boundary component of $R$. This fact can be proved in the quite same way as for a $(\widetilde{P}) L_{1}$-principal function $f_{1}$. But all the functions $f_{0}-f_{1}$, where $f_{0}$ and $f_{1}$ have the same singularities, are constant if and only if $R \in O_{K D}$ (Ahlfors-Sario [1]). Therefore there is a function $f_{0}$ on $R$ which is not a $(\widetilde{P}) L_{1}$-principal function, but can be extended continuously on a Kerékjártó-Stoilow compactification.

\section{REFERENCES}

1. L. V. Ahlfors, and L. Sario, Riemann surfaces, Princeton, 1960.

2. C. Constantinescu and A. Cornea, Idealeränder Riemannscher Flächen, Springer, 1963.

3. M. Jurchescu, Modulus of a boundary component, Pacific J. Math. 8 (1958), 791809.

4. Y. Kusunoki, Theory of Abelian integrals and its applications to conformal mappings, Mem. Coll. Sci. Univ. Kyoto, Ser. A. Math. 32 (1959), 235-258.

5. Characterizations of canonical differentials, J. Math. Kyoto Univ. 5 (1966), 197-207.

6. M. Mori, On the semi-exact canonical differentials of the first kind, Proc. Japan Acad. 36 (1960), 252-257.

7. — Canonical conformal mappings of open Riemann surfaces, J. Math. Kyoto Univ. 3 (1964), 169-192.

8. M. Nakai and L. Sario, Construction of principal functions by orthogonal projection, Canad. J. Math. 18 (1966), 887-896.

9. B. Rodin and L. Sario, Convergence of normal operators, Kôdai Math. Sem. Rep. 19 (1967), 165-173.

10. L. Sario, A linear operator method on arbitrary Riemann surfaces, Trans. Amer. Math. Soc. 72 (1952), 281-295.

Received November 9, 1967. Work supported in part by Contract N0014-67-A-01120015 at Stanford University.

Kyoto UNIVERSITY AND STANFORD UNIVERSITY 



\section{PACIFIC JOURNAL OF MATHEMATICS}

\section{EDITORS}

\author{
H. ROYDEN \\ Stanford University \\ Stanford, California \\ Richard Pierce \\ University of Washington \\ Seattle, Washington 98105
}

\author{
J. DugundJI \\ Department of Mathematics \\ University of Southern California \\ Los Angeles, California 90007 \\ BASIL GoRDON \\ University of California \\ Los Angeles, California 90024
}

\section{ASSOCIATE EDITORS}
E. F. BECKENBACH
B. H. Neumann
F. WOLF
K. YoSHIDA

\section{SUPPORTING INSTITUTIONS}

\author{
UNIVERSITY OF BRITISH COLUMBIA \\ CALIFORNIA INSTITUTE OF TECHNOLOGY \\ UNIVERSITY OF CALIFORNIA \\ MONTANA STATE UNIVERSITY \\ UNIVERSITY OF NEVADA \\ NEW MEXICO STATE UNIVERSITY \\ OREGON STATE UNIVERSITY \\ UNIVERSITY OF OREGON \\ OSAKA UNIVERSITY \\ UNIVERSITY OF SOUTHERN CALIFORNIA
}

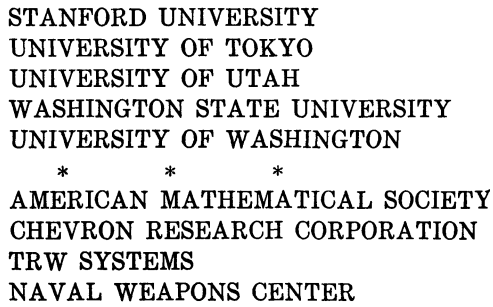

The Supporting Institutions listed above contribute to the cost of publication of this Journal, but they are not owners or publishers and have no responsibility for its content or policies.

Mathematical papers intended for publication in the Pacific Journal of Mathematics should be in typed form or offset-reproduced, double spaced with large margins. Underline Greek letters in red, German in green, and script in blue. The first paragraph or two must be capable of being used separately as a synopsis of the entire paper. It should not contain references to the bibliography. Manuscripts, in duplicate if possible, may be sent to any one of the four editors. Please classify according to the scheme of Math. Rev. 36, 1539-1546. All other communications to the editors should be addressed to the managing editor, Richard Arens, University of California, Los Angeles, California, 90024.

50 reprints are provided free for each article; additional copies may be obtained at cost in multiples of 50 .

The Pacific Journal of Mathematics is published monthly. Effective with Volume 16 the price per volume (3 numbers) is $\$ 8.00$; single issues, $\$ 3.00$. Special price for current issues to individual faculty members of supporting institutions and to individual members of the American Mathematical Society: $\$ 4.00$ per volume; single issues $\$ 1.50$. Back numbers are available.

Subscriptions, orders for back numbers, and changes of address should be sent to Pacific Journal of Mathematics, 103 Highland Boulevard, Berkeley, California, 94708.

PUBLISHED BY PACIFIC JOURNAL OF MATHEMATICS, A NON-PROFIT CORPORATION

Printed at Kokusai Bunken Insatsusha (International Academic Printing Co., Ltd.), 7-17, Fujimi 2-chome, Chiyoda-ku, Tokyo, Japan. 


\section{Pacific Journal of Mathematics}

\section{Vol. 31, No. $2 \quad$ December, 1969}

Efraim Pacillas Armendariz, Quasi-injective modules and stable torsion

classes..........................................

J. Adrian (John) Bondy, On Ulam's conjecture for separable graphs...

Vasily Cateforis and Francis Louis Sandomierski, On commutative rings over which the singular submodule is a direct summand for every module .....

Rafael Van Severen Chacon, Approximation of transformations with continuous

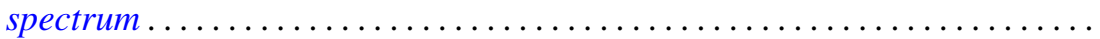

Raymond Frank Dickman and Alan Zame, Functionally compact spaces ...... 303

Ronald George Douglas and Walter Rudin, Approximation by inner

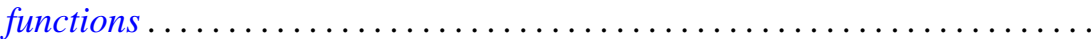

John Walter Duke, A note on the similarity of matrix and its conjugate

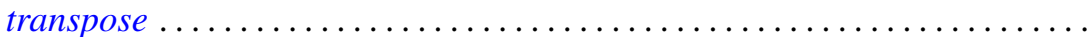

Micheal Neal Dyer and Allan John Sieradski, Coverings of mapping

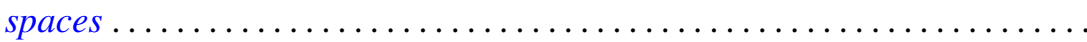

Donald Campbell Dykes, Weakly hypercentral subgroups of finite groups .....

Nancy Dykes, Mappings and realcompact spaces.....................

Edmund H. Feller and Richard Laham Gantos, Completely injective

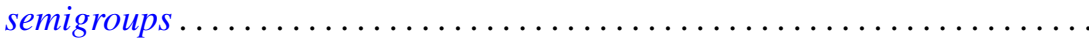

Irving Leonard Glicksberg, Semi-square-summable Fourier-Stieltjes

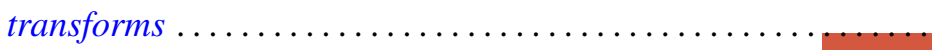

Samuel Irving Goldberg and Kentaro Yano, Integrability of almost cosymplectic structures...

Seymour Haber and Charles Freeman Osgood, On the sum $\sum\langle n \alpha\rangle^{-t}$ and numerical integration ..........................

Sav Roman Harasymiv, Dilations of rapidly decreasing functions ....

William Leonard Harkness and R. Shantaram, Convergence of a sequence of

transformations of distribution functions

Herbert Frederick Kreimer, Jr., A note on the outer Galois theory of rings ...

James Donald Kuelbs, Abstract Wiener spaces and applications to analysis. .

Roland Edwin Larson, Minimal $T_{0}$-spaces and minimal $T_{D}$-spaces...

A. Meir and Ambikeshwar Sharma, On Ilyeff's conjecture .

Isaac Namioka and Robert Ralph Phelps, Tensor products of compact convex sets....

James L. Rovnyak, On the theory of unbounded Toeplitz operators ....

Benjamin L. Schwartz, Infinite self-interchange graphs.......

George Szeto, On the Brauer splitting theorem...

Takayuki Tamura, Semigroups satisfying identity $x y=f(x$,

Kenneth Tolo, Factorizable semigroups .................. 